\title{
Variations on themes of Kostant
}

\author{
Victor Ginzburg
}

To Bert Kostant with admiration

\begin{abstract}
Let $\mathfrak{g}$ be a complex semisimple Lie algebra, and let ${ }^{L} G$ be a complex semisimple group with trivial center whose root system is dual to that of $\mathfrak{g}$. We establish a graded algebra isomorphism $H^{\bullet}\left(X_{\lambda}, \mathbb{C}\right) \cong \mathrm{Sg}^{e} / I_{\lambda}$, where $X_{\lambda}$ is an arbitrary spherical Schubert variety in the loop Grassmannian for ${ }^{L} G$, and $I_{\lambda}$ is an appropriate ideal in the symmetric algebra of $\mathfrak{g}^{e}$, the centralizer of a principal nilpotent in $\mathfrak{g}$.

We also discuss a 'topological' proof of Kostant's result on the structure of $\mathbb{C}[\mathfrak{g}]$.
\end{abstract}

\section{Cohomology of 'spherical' Schubert varieties}

In this paper, we discuss a few geometric results which were, to a great extent, inspired by three fundamental papers of Bertram Kostant [Ko1]-[Ko3].

1.1 Let $\mathfrak{g}$ be a complex semisimple Lie algebra and write $\mathfrak{g}^{x} \subset \mathfrak{g}$ for the centralizer of an element $x \in \mathfrak{g}$. We fix a principal $\mathfrak{s l}_{2}$-triple $\langle h, e, f\rangle \subset \mathfrak{g}$. Thus, $\mathfrak{h}:=\mathfrak{g}^{h}$ is a Cartan subalgebra of $\mathfrak{g}$. The element $e \in \mathfrak{g}$ is a principal nilpotent and we make the choice of positive roots of $(\mathfrak{g}, \mathfrak{h})$ so that $e$ is contained in the span of simple root vectors.

We write $\mathfrak{h}_{\mathbb{Z}}^{*} \subset \mathfrak{h}^{*}$ for the weight lattice of $(\mathfrak{g}, \mathfrak{h})$. Given an finite dimensional $\mathfrak{g}$-module $V$ and a weight $\mu \in \mathfrak{h}_{\mathbb{Z}}^{*}$, let $V(\mu) \subset V$ denote the corresponding $\mu$-weight space, let Spec $V \subset \mathfrak{h}_{\mathbb{Z}}^{*}$ be the set formed by the weights which occur in $V$ with nonzero multiplicity, ie. such that $V(\mu) \neq 0$. We ignore weight multiplicities and let $I(V) \subset \mathbb{C}\left[\mathfrak{h}^{*}\right]$ denote the ideal of polynomials vanishing at the set Spec $V_{\lambda}$, viewed as a finite reduced subscheme in $\mathfrak{h}^{*}$. Thus, the coordinate ring $\mathbb{C}\left[\operatorname{Spec} V_{\lambda}\right]=\mathbb{C}\left[\mathfrak{h}^{*}\right] / I(V)$ is a finite dimensional algebra.

Write Uk, resp. Sk, for the universal enveloping, resp. symmetric, algebra of a Lie algebra $\mathfrak{k}$. A filtration on $\mathfrak{k}$ gives rise to a filtration on $\mathrm{Uk}^{\mathfrak{k}}$, resp. on $\mathrm{Sk}$, not to be confused with the standard increasing filtration on an enveloping algebra.

Following B. Kostant and R. Brylinski, on $\mathfrak{h}$, one introduces an increasing filtration $F . \mathfrak{h}$, where $F_{k} \mathfrak{h}:=\left\{x \in \mathfrak{h} \mid \operatorname{ad}^{k+1} e(x)=0\right\}, k=0,1, \ldots$ The induced filtration gives Uh the structure of a nonnegatively filtered algebra. Clearly, one has $U \mathfrak{h}=S \mathfrak{h}=\mathbb{C}\left[\mathfrak{h}^{*}\right]$. In particular, we may (and will) view $\mathbb{C}\left[\operatorname{Spec} V_{\lambda}\right]$ as a quotient of the algebra Uh. In this way, the algebra $\mathbb{C}\left[\operatorname{Spec} V_{\lambda}\right]$ acquires an increasing filtration that descends from the KostantBrylinski filtration on $U \mathfrak{h}$. For the associated graded algebras, we have $\operatorname{gr}^{F} \mathbb{C}\left[\operatorname{Spec} V_{\lambda}\right]=$ $\operatorname{gr}^{F} \mathrm{Uh} / \operatorname{gr}^{F} I(V)$. 
1.2 Write ${ }^{L} G$ for a split connected semisimple group (called Langlands dual group) that has trivial center, i.e., is of adjoint type, and that has a maximal torus, ${ }^{L} T \subset{ }^{L} G$, such that, we have $\operatorname{Lie}^{L} T=\mathfrak{h}^{*}$ and the root system of $\left({ }^{L} G,{ }^{L} T\right)$ is dual to that of $\mathfrak{g}$. Let $W$ denote the Weyl group, the same both for $(\mathfrak{g}, \mathfrak{h})$ and for $\left({ }^{L} G,{ }^{L} T\right)$.

Let $\mathbb{C}((z))$ be the field of formal Laurent series and $\mathbb{C}[[z]] \subset \mathbb{C}((z))$ its ring of integers, the ring of formal power series. Let ${ }^{L} G((z))$, resp. ${ }^{L} G[[z]]$, denote the group of $\mathbb{C}((z))$-rational, resp. $\mathbb{C}[[z]]$-rational, points of ${ }^{L} G$. The coset space $\mathrm{Gr}:={ }^{L} G((z)) /{ }^{L} G[[z]]$ is called the loop Grassmannian.

The space Gr has a natural structure of an ind-scheme, a direct limit of an ascending chain of projective varieties of increasing dimension, see e.g. [G1, §1.2] or [Lu] for an elementary, and [BD] for a much more elaborate treatment. There are canonical bijections

$$
\mathrm{Gr}^{{ }^{L} T}=\operatorname{Hom}_{\text {alg. group }}\left(\mathbb{C}^{\times},{ }^{L} T\right)=\mathfrak{h}_{\mathbb{Z}}^{*}\left(\subset \mathfrak{h}^{*}\right) .
$$

Here, the space on the left denotes the fixed point set of the left ${ }^{L} T$-action on $\mathrm{Gr}$, and the Hom-space in the middle denotes the coweight lattice of ${ }^{L} T$. Let $\mathrm{Gr}_{\lambda}$ denote the ${ }^{L} G[[z]]$-orbit of the ${ }^{L} T$-fixed point corresponding to such a coweight $\lambda$. In this way, one obtains a bijection between the set of ${ }^{L} G[[z]]$-orbits in $G r$ and the set $\mathfrak{h}_{\mathbb{Z}}^{*} / W$, of the Weyl group orbits in $\mathfrak{h}_{\mathbb{Z}}^{*}$. The closure, $\overline{G r}_{\lambda} \subset G r$, to be referred to as 'spherical Schubert variety', is known to be a finite dimensional projective variety, singular in general.

1.3 For any Lie algebra $\mathfrak{k}$, a $\mathfrak{k}$-module $V$, and a subset $S \subset V$, resp. $J \subset \mathrm{Uk}$, we put

$$
\operatorname{Ann}[\mathfrak{k} ; S]:=\{u \in \mathrm{Uk} \mid u(s)=0 \forall s \in S\}, \quad \text { resp. } V^{J}:=\{v \in V \mid u(v)=0 \forall u \in J\} .
$$

Given an anti-dominant weight $\lambda \in \mathfrak{h}_{\mathbb{Z}}^{*}$, let $V_{\lambda}$ be an irreducible finite dimensional $\mathfrak{g}$-representation with lowest weight $\lambda$, and let $v_{\lambda} \in V_{\lambda}$ be a lowest weight vector.

The centralizer of $e$ is an ad $h$-stable abelian Lie subalgebra $\mathfrak{g}^{e} \subset \mathfrak{g}$. The grading induced by the ad $h$-action makes $\mathrm{Ug}^{e}$ a nonnegatively graded commutative algebra. It is clear that $\operatorname{Ann}\left[\mathfrak{g}^{e} ; v_{\lambda}\right]$, the annihilator of the element $v_{\lambda}$, is a graded ideal in $\mathrm{Ug}^{e}$. Thus, the quotient $\mathrm{Ug}^{e} / \operatorname{Ann}\left[\mathfrak{g}^{e} ; v_{\lambda}\right]$ is a graded finite dimensional commutative algebra.

In this paper, we write $H^{\bullet}(-)$ for singular cohomology with $\mathbb{C}$-coefficients. One of our main results reads

Theorem 1.3.2. The $\mathrm{Ug}^{e}$-action on $V_{\lambda}$ factors through the quotient $\mathrm{Ug}^{e} / \operatorname{Ann}\left[\mathfrak{g}^{e} ; v_{\lambda}\right]$, and there are natural graded algebra isomorphisms

$$
H^{\bullet}\left(\overline{\mathrm{Gr}}_{\lambda}\right) \simeq \mathrm{Ug}^{e} / \operatorname{Ann}\left[\mathfrak{g}^{e} ; v_{\lambda}\right] \simeq \operatorname{gr}^{F} \mathbb{C}\left[\operatorname{Spec} V_{\lambda}\right]
$$

Remarks 1.3.4. (i) The isomorphism on the left of (1.3.3) was communicated to me, as a conjecture, by Dale Peterson in the Summer of 1997. This isomorphism is induced by the restriction map $H^{\bullet}(\mathrm{Gr}) \rightarrow H^{\bullet}\left(\overline{\mathrm{Gr}}_{\lambda}, \mathbb{C}\right)$, using a natural graded algebra isomorphism $H^{\bullet}(\mathrm{Gr}) \simeq \mathrm{Ug}^{e}$, proved in [G1, Proposition 1.7.2].

(ii) The isomorphism on the right of (1.3.3) is induced by the Kostant-Brylinski graded algebra isomorphism, $\operatorname{gr}^{F} \mathrm{Uh} \cong \mathrm{Ug}^{e}$, cf. [Bry]. In other words, the second isomorphism in (1.3.3) amounts to an equality $\operatorname{gr}^{F} I\left(V_{\lambda}\right)=\operatorname{Ann}\left[\mathfrak{g}^{e} ; v_{\lambda}\right]$, of graded ideals in $\mathrm{Ug}^{e}$. 
(iii) The composite isomorphism in (1.3.3) provides a description of the cohomology of $\overline{\mathrm{Gr}}_{\lambda}$ similar to the description of the cohomology of the flag variety $G / B$, due to Kostant. Such a description was furher extended to the case of arbitrary Schubert varieties in $G / B$ by Akyildiz and Carrell [AC]. It would be interesting to obtain a direct proof of our isomorphisms using the techniques of $\mathrm{AC}$, at least in the simply laced case.

(iv) There is an analogue of Theorem 1.3 .2 for an arbitrary, not necessarily spherical Schubert variety $X_{\lambda} \subset \mathrm{Gr}$. Specifically, in [GK], $\S 4.3$, the authors have associated with any, not necessarily anti-dominant, weight $\lambda \in \mathfrak{h}_{\mathbb{Z}}^{*}$, a certain finite dimensional graded $\mathrm{Ug}^{e}$ module $F_{\lambda}$. The module $F_{\lambda}$ was defined in terms of quantum group cohomology, and it was conjectured that the same module has an alternate geometric interpretation in terms of intersection cohomology of the Schubert variety $X_{\lambda}$. That conjecture was later proved in [ABG], Theorem 10.2.3. Using these results, it is straightforward to extend our proof of Theorem 1.3.2 to obtain an algebra isomorphism of the form $H^{\bullet}\left(X_{\lambda}\right) \cong \mathrm{Ug}^{e} / \operatorname{Ann}\left[\mathfrak{g}^{e} ; F_{\lambda}\right]$.

1.4 From Theorem 1.3.2, comparing dimensions of $\mathbb{C}\left[\operatorname{Spec} V_{\lambda}\right]$ and of $V_{\lambda}$, we deduce

Corollary 1.4.1. One has $V_{\lambda}=\mathrm{Ug}^{e} \cdot v_{\lambda}$ if and only if any weight occurs in $V_{\lambda}$ with multiplicity $\leq 1$.

We remark that there are very few representations, besides the minuscule ones, with all weights of multiplicity at most one. For simple groups, these are: $\mathrm{S}^{n}\left(\mathbb{C}^{n}\right)$ for $G=S L(n)$; the standard $2 n+1$-dimensional representation for $G=S O(2 n+1)$, and a 7-dimensional representation for a group $G$ of type $G_{2}$.

Let $Q(\mathfrak{g}, \mathfrak{h}) \subset \mathfrak{h}_{\mathbb{Z}}^{*}$ be the root lattice. In the special case where $\lambda \in Q(\mathfrak{g}, \mathfrak{h})$, Corollary 1.4.1 is equivalent to the following unpublished result of B. Kostant.

Proposition 1.4.2. Let $\lambda \in Q(\mathfrak{g}, \mathfrak{h})$ be a anti-dominant weight. Then, $V_{\lambda}=\mathrm{Ug}^{e} \cdot v_{\lambda}$ holds if and only if $\operatorname{dim} V_{\lambda}(0)=1$.

Proof. 11 Fix an arbitrary anti-dominant weight $\lambda$ contained in the root lattice of $\mathfrak{g}$ and an $h$-stable direct sum decomposition $V_{\lambda}=\mathfrak{g}^{e}\left(V_{\lambda}\right) \oplus M$. We claim that

$$
V_{\lambda}=\mathrm{Ug}^{e} \cdot M \quad \text { and } \quad \operatorname{dim} M=\operatorname{dim} V_{\lambda}(0) .
$$

To prove the first equation, assume $v \in V_{\lambda}$ is a nonzero vector that does not belong to $\mathrm{Ug}^{e} \cdot M$. We may assume without loss of generality that $v$ is an $h$-eigenvector with an integral eigenvalue $c$. Furthermore, let $v$ be such that $c$ is the minimal possible among those arising in this way for various $h$-eigenvectors $v$ as above.

Now, since $V_{\lambda}=\mathfrak{g}^{e}\left(V_{\lambda}\right) \oplus M$, we have $v=\sum_{j} a_{j} \cdot v_{j}+m$, for some ad $h$-weight vectors $a_{j} \in \mathfrak{g}^{e}$ and some $h$-eigenvectors $m \in M, v_{j} \in V_{\lambda}$, such that $v_{j} \notin \mathrm{Ug}^{e} \cdot M$ for at least one $j$. Write $c_{j} \in \mathbb{Z}$ for the $h$-eigenvalue of the vector $v_{j}$. The weights of the ad $h$-action on $\mathfrak{g}^{e}$ being positive integers (equal to twice the exponents of $\mathfrak{g}^{e}$ ), we deduce that $c_{j}<c$. Hence, the minimality of $c$ forces $v_{j}=0$ for all $j$. Thus, $v=m \in M$. This is a contradiction, and the first equation in (1.4.3) follows.

\footnotetext{
${ }^{1}$ We are indebted to B. Kostant for kindly communicating to us both the statement and the main idea of the short direct prooof of this result reproduced below.
} 
To prove the second equation, let $V_{\lambda}^{*}$ denote the contragredient representation. By a result of Kostant, cf. Theorem 1.6.1(ii) below, one has $\operatorname{dim}\left(V_{\lambda}^{*}\right)^{\mathfrak{g}^{e}}=\operatorname{dim}\left(V_{\lambda}^{*}\right)(0)=$ $\operatorname{dim} V_{\lambda}(0)$. Furthermore, the annihilator of the subspace $\left(V_{\lambda}^{*}\right)^{\mathfrak{g}^{e}}$ with respect to the canonical perfect pairing $V_{\lambda} \times V_{\lambda}^{*} \rightarrow \mathbb{C}$ equals $\mathfrak{g}^{e}\left(V_{\lambda}\right) \subset V_{\lambda}$. Thus, we deduce $\operatorname{dim} \mathfrak{g}^{e}\left(V_{\lambda}\right)+$ $\operatorname{dim} V_{\lambda}(0)=\operatorname{dim} V_{\lambda}$, and the second equation in (1.4.3) follows.

Let finally $\operatorname{dim} V_{\lambda}(0)=1$. Observe that $v_{\lambda} \notin \mathfrak{g}^{e}\left(V_{\lambda}\right)$ so, by the dimension formula in (1.4.3), the space $M=\mathbb{C} \cdot v_{\lambda}$ provides, in this case, a direct complement to $\mathfrak{g}^{e}\left(V_{\lambda}\right)$. Then, the first equation in (1.4.3) yields $V_{\lambda}=\mathrm{Ug}^{e} \cdot v_{\lambda}$. This proves one implication; the proof of the opposite implication is similar and is left to the reader.

We note that the dimension equality in (1.4.3) clearly holds in the setting of Corollory 1.4.1 as well. However, the proof of Proposition 1.4.2 does not go through since the equation $\operatorname{dim} V_{\lambda}^{e}=\operatorname{dim} V_{\lambda}(0)$ fails unless $\lambda \in Q(\mathfrak{g}, \mathfrak{h})$.

1.5 We will use simplified notation $\mathrm{Sh}^{W}$ for the algebra $(\mathrm{Sh})^{W}=\mathbb{C}\left[\mathfrak{h}^{*}\right]^{W} \cong \mathbb{C}\left[\operatorname{Lie}^{L} T\right]^{W}$. Let $\mathfrak{m}_{o}:=\mathrm{Sh}_{+}^{W}$ denote the augmentation ideal of $\mathrm{Sh}^{W}$. Given a weight $\lambda$, write $W_{\lambda} \subset W$ for the isotropy group of $\lambda$, and let $P_{\lambda} \subset{ }^{L} G$ be the corresponding parabolic subgroup. Thus, one has graded algebra imbeddings $\mathrm{Sh}^{W} \hookrightarrow \mathrm{Sh}^{W_{\lambda}} \hookrightarrow \mathrm{Sh}$.

Further, view $\lambda$ as a ${ }^{L} T$-fixed point in the loop Grassmannian. The corresponding ${ }^{L} G$-orbit, ${ }^{L} G \cdot \lambda \cong{ }^{L} G / P_{\lambda}$, is a partial flag manifold. In this way, we get a chain of imbeddings

$$
{ }^{L} G / P_{\lambda}={ }^{L} G \cdot \lambda \longleftrightarrow{ }^{\imath_{\lambda}} \longrightarrow{ }^{L} G[[z]] \cdot \lambda=\mathrm{Gr}_{\lambda} \stackrel{\jmath_{\lambda}}{\longrightarrow} \overline{G r}_{\lambda}
$$

where the map $\imath_{\lambda}$ is a homotopy equivalence and the map $\jmath_{\lambda}$ is an open imbedding. Therefore, the composite map in (1.5.1) induces, via Theorem 1.3.2, graded algebra morphisms

$$
\mathrm{U} \mathfrak{g}^{e} / \operatorname{Ann}\left[\mathfrak{g}^{e} ; v_{\lambda}\right]=H \cdot\left(\overline{\mathrm{Gr}}_{\lambda}\right) \stackrel{\left(\jmath_{\lambda} \circ \imath_{\lambda}\right)^{*}}{\longrightarrow} H^{\bullet}\left({ }^{L} G / P_{\lambda}\right)=\mathrm{Sh}^{W_{\lambda}} / \mathfrak{m}_{o} \cdot \mathrm{Sh}^{W_{\lambda}},
$$

where the last equality is the Borel isomorphism.

Proposition 1.5.3. For any anti-dominant weight $\lambda$, the map (1.5.2) is surjective. Moreover, it may be factored naturally as a composite

$$
\mathrm{U} \mathfrak{g}^{e} / \operatorname{Ann}\left[\mathfrak{g}^{e} ; v_{\lambda}\right] \stackrel{u \mapsto u\left(v_{\lambda}\right)}{\longrightarrow} V_{\lambda} \stackrel{f_{o}}{\longrightarrow} \mathrm{Sh}^{W_{\lambda}} / \mathfrak{m}_{o} \cdot \mathrm{Sh}^{W_{\lambda}} .
$$

Friedman and Morgan [FM], have introduced a certain linear map $f: V_{\lambda} \rightarrow \mathrm{Sh}^{W_{\lambda}}$, and conjectured that the composite proj。 $f: V_{\lambda} \rightarrow \mathrm{Sh}^{W_{\lambda}} \rightarrow \mathrm{Sh}^{W_{\lambda}} / \mathfrak{m}_{o} \cdot \mathrm{Sh}^{W_{\lambda}}$, is a surjective map. It is not difficult to verify, going through the constructions of the maps in question, that one actually has $f_{o}=\operatorname{proj} \circ f$. Thus, our proof of Proposition 1.5.3, to be given in 3.3 , yields at the same time a proof of (a strengthening of) the Friedman-Morgan conjecture.

1.6 We recall that the orbit $\mathrm{Gr}_{\mu}$ is closed in $\mathrm{Gr}$ if and only if the weight $\mu$ is either minuscule or zero. In such a case, each of the imbeddings in (1.5.1) is an equality and the restriction map $\left(\iota_{\mu}^{\circ} \jmath_{\mu}\right)^{*}$, in (1.5.2), becomes an isomorphism. 
Theorem 1.6.1. Let $\mu$ be either a minuscule anti-dominant weight or zero. Then,

(i) Each of the two maps in (1.5.4) is a bijection, and the composite yields a graded algebra isomorphism $\mathrm{Ug}^{e} / \operatorname{Ann}\left[\mathfrak{g}^{e} ; v_{\mu}\right] \cong \mathrm{Sh}^{W_{\mu}} / \mathfrak{m}_{o} \cdot \mathrm{Sh}^{W_{\mu}}$.

(ii) For any finite dimensional $\mathfrak{g}$-module $V$ such that $\operatorname{Spec} V \subset \mu+Q(\mathfrak{g}, \mathfrak{h})$, we have

$$
\operatorname{dim} V^{\operatorname{Ann}\left[\mathfrak{g}^{e} ; v_{\mu}\right]} /|W|=\operatorname{dim} V(\mu) /\left|W_{\mu}\right| .
$$

The bijectivity of the first map in (1.5.4) for a minuscule weight $\mu$ is immediate from Corollary 1.4.1, see also [G1], Proposition 1.9, Proposition 1.8.1. The bijectivity of the second map $f_{o}$ will be proved in $\$ 3.5$. It has also been established earlier by Friedman and Morgan [FM], in a totally different way.

Remarks 1.6.2. (1) For $\mu=0$, the statement of Theorem 1.6.1(ii) is a classic result of Kostant Ko1, cf. also Theorem 4.1.1 below. Note that this special case of Theorem 1.6.1(ii) immediately implies, by a standard semicontinuity argument, another important result of Kostant saying that the function $x \mapsto \operatorname{dim} V^{\mathfrak{g}^{x}}$ is constant on the set of regular elements of $\mathfrak{g}$, whenever $\operatorname{Spec} V \subset Q(\mathfrak{g}, \mathfrak{h})$.

(2) An alternative much more elementary proof of the graded space isomorphism from Theorem 1.6.1(i) is due to B. Gross [Gr, Proposition 6.4. The technique of loc. cit. was insufficient, however, for establishing the isomorphism of algebras.

ACKnowledgements. I am grateful to Dale Peterson and Bert Kostant for interesting discussions, and also to Mark Reeder for several very helpful comments on an earlier draft of the paper, and to Braham Broer for bringing reference $\mathrm{Bro}$ to my attention.

\section{$2 \quad$ A geometric result}

Our proof of Theorem 1.3.2 is based on a simple general result concerning intersection cohomology which we are going to state and prove below. Write $\otimes=\otimes_{\mathbb{C}}$.

2.1 Let $Y$ be a (not necessarily smooth) complex algebraic variety, and $D^{b}(Y)$ the bounded derived category of constructible complexes of $\mathbb{C}$-sheaves on $Y$. Let $\mathbb{C}_{Y} \in D^{b}(Y)$ be the constant sheaf, resp. $I C(Y) \in D^{b}(Y)$ be the intersection cohomology complex on $Y$ i.e., the 'intermediate extension' of the constant sheaf on a Zariski open smooth subset $Y_{0} \subset Y$. In this paper, we will use the 'naive' normalization in which the restriction of $I C(Y)$ to $Y_{0}$ is a constant sheaf concentrated in degree 0 (not in degree $-\operatorname{dim} Y$ as in [BBD]). Thus non-zero cohomology sheaves, $\mathcal{H}^{i} I C(Y)$, may occur only in degrees $0 \leq i \leq \operatorname{dim} Y$. We write $I H^{\bullet}(Y)=H^{\bullet}(Y, I C(Y))$, for the intersection cohomology groups of $Y$ with complex coefficients.

We assume $Y$ is irreducible. Then, in degree zero, we have $\mathbb{C}=H^{0}(Y)=I H^{0}(Y)=$ $\operatorname{Hom}_{D^{b}(Y)}\left(\mathbb{C}_{Y}, I C(Y)\right)$. Therefore, there exists a nonzero morphism $\kappa: \mathbb{C}_{Y} \rightarrow I C(Y)$, unique up to a constant factor. This gives the induced map of (hyper-) cohomology,

$$
\kappa_{*}: H^{\bullet}(Y)=H^{\bullet}\left(Y, \mathbb{C}_{Y}\right) \longrightarrow H^{\bullet}(Y, I C(Y))=I H^{\bullet}(Y) \text {. }
$$


2.2 Let $Y$ be an irreducible projective variety with an algebraic stratification $Y=$ $\sqcup_{s \in \mathcal{S}} Y_{s}$. The strata $Y_{s}$ are assumed to be smooth locally closed subvarieties labelled by the elements of a finite set $\mathcal{S}$.

We will use the standard yoga of weights introduced by Deligne [De], cf. also [BBD]. Recall that a mixed complex $A$ on $Y$ is said to be pointwise pure if $i_{y}^{*} A$ is pure for any $y \in Y$, where $i_{y}:\{y\} \hookrightarrow Y$ denotes the imbedding.

Our geometric result about intersection cohomology reads

Proposition 2.2.1. Assume the following two conditions hold:

- The complex $I C(Y)$ is pointwise pure;

- Each stratum $Y_{s}$ is isomorphic to an affine (e.g. vector) bundle over a smooth connected, and simply-connected projective variety.

Then, the map $\kappa_{*}$ in (2.1.1) is injective, equivalently, the dual map $I^{\bullet}(Y) \rightarrow H .(Y)$, to homology, is surjective.

Proof. For each $s \in \mathcal{S}$, write $\bar{Y}_{s}$ for the closure of $Y_{s}$ and let $j_{s}: Y_{s} \hookrightarrow Y$, resp. $j_{s}^{\prime}: Y_{s} \hookrightarrow$ $\bar{Y}_{s}$, denote the natural inclusion. Then, for any $A \in D^{b}(Y)$, there is a standard convergent spectral sequence of the form

$$
E_{2}^{p, q}(A)=\bigoplus_{\operatorname{dim} Y_{s}=-p} H^{p+q}\left(\bar{Y}_{s},\left(j_{s}^{\prime}\right) ! j_{s}^{*} A\right) \Rightarrow E_{\infty}^{p, q}(A)=\operatorname{gr}_{p} H^{p+q}(Y, A)
$$

where in the last equality on the right we have used that $H_{c}^{\bullet}(Y, A)=H^{\bullet}(Y, A)$ since $Y$ is compact.

First, let $A=\mathbb{C}_{Y}$. Then, $j_{s}^{*} A=\mathbb{C}_{Y_{s}}$, hence, we have $H^{n}\left(\bar{Y}_{s},\left(j_{s}^{\prime}\right) ! j_{s}^{*} A\right)=H_{c}^{n}\left(Y_{s}\right)$, the cohomology of $Y_{s}$ with compact support. By the second assumption of the proposition, the cohomology $H_{c}^{\cdot}\left(Y_{s}\right)$ is pure. It follows that, for $A=\mathbb{C}_{Y}$, the corresponding spectral sequence (2.2.2) degenerates at the $E_{2}$-term, by a standard argument due to Deligne. Thus, we obtain $E_{2}\left(\mathbb{C}_{Y}\right)=E_{\infty}\left(\mathbb{C}_{Y}\right)=\operatorname{gr} H^{\bullet}(Y)$.

Next, we let $A=I C(Y)$. Then, each cohomology sheaf $\mathcal{H}^{p} j_{s}^{*} A$ is a locally constant, hence constant, sheaf on $Y_{s}$, for any $s \in \mathcal{S}$. Furthermore, the pointwise purity assumption on $I C(Y)$ insures that each of the local systems $\mathcal{H}^{p} j_{s}^{*} I C(Y)$ is pure. Hence, the spectral sequence (2.2.2) for $A=I C(Y)$ degenerates at the $E_{2}$-term again. Thus, we obtain $E_{2}(I C(Y))=E_{\infty}(I C(Y))=\operatorname{gr} I H^{\bullet}(Y)$.

Now, the canonical sheaf morphism $\kappa: \mathbb{C}_{Y} \rightarrow I C(Y)$ induces a morphism of spectral sequences associated with $\mathbb{C}_{Y}$ and with $I C(Y)$, respectively. For the corresponding $E_{2^{-}}$ terms, the resulting map $E_{2}^{p, q}\left(\mathbb{C}_{Y}\right) \rightarrow E_{2}^{p, q}(I C(Y))$ splits into a direct sum of the form

$$
\bigoplus_{\operatorname{dim} Y_{s}=-p}\left[H_{c}^{p+q}\left(Y_{s}, \mathbb{C}_{Y_{s}}\right) \stackrel{\kappa_{s}}{\longrightarrow} H_{c}^{p+q}\left(Y_{s}, \mathcal{H}^{\bullet} j_{s}^{*} I C(Y)\right)\right]
$$

Here, $\kappa_{s}$ is a map induced by the morphism of sheaves $\left.\kappa\right|_{Y_{s}}: \mathbb{C}_{Y_{s}} \rightarrow \mathcal{H}^{0} j_{s}^{*} I C(Y)$, the restriction of the canonical morphism $\kappa: \mathbb{C}_{Y} \rightarrow \mathcal{H}^{0} I C(Y)$ to $Y_{s}$. Since $\kappa$ is clearly injective, the restriction of $\kappa$ to $Y_{s}$ is injective as well. It follows that each of the maps $\kappa_{s}$, hence the map (2.2.3) itself, is injective. 
Since both spectral sequences degenerate at the corresponding $E_{2}$-terms, we conclude that we have proved injectivity of the map

$$
E_{2}\left(\mathbb{C}_{Y}\right)=\operatorname{gr} H^{\bullet}\left(Y, \mathbb{C}_{Y}\right) \longrightarrow E_{2}(I C(Y))=\operatorname{gr} I H^{\bullet}(Y) .
$$

The latter map is nothing but the map gr $\kappa_{*}$, the associated graded map for the morphism (2.1.1). Thus, the injectivity of gr $\kappa_{*}$ implies injectivity of the map $\kappa_{*}$ itself.

2.3 Equivariant setting. Applications of Proposition 2.2.1 often involve varieties with a group action. Let $\mathbb{G}$ be a complex connected reductive group and $p t:=$ point.

Given a $\mathbb{G}$-variety $Y$, there is a standard functor $H_{\mathbb{G}}^{\cdot}(Y,-)$, of $\mathbb{G}$-equivariant cohomology, see [BL1]. This functor assigns to a $\mathbb{G}$-equivariant constructible complex $\mathcal{L}$, on $Y$, a graded $H_{\mathbb{G}}(p t)$-module $H_{\mathbb{G}}(Y, \mathcal{L})$, the total $\mathbb{G}$-equivariant (hyper-) cohomology group of $\mathcal{L}$. In the special case of the constant sheaf $\mathbb{C}_{Y}$, we have $H_{\mathbb{G}}^{\cdot}\left(Y, \mathbb{C}_{Y}\right)=H_{\mathbb{G}}(Y)$, the $\mathbb{G}$-equivariant cohomology of the space $Y$. This is a graded $H_{\mathbb{G}}^{\cdot}(p t)$-algebra; moreover, it is a finite rank free graded $H_{\mathbb{G}}(p t)$-module provided $Y$ has a $\mathbb{G}$-stable stratification $Y=\sqcup_{s \in \mathcal{S}} Y_{s}$, where each stratum $Y_{s}$ is (equivariantly) isomorphic to a $\mathbb{G}$-equivariant affine bundle on a smooth projective $\mathbb{G}$-variety.

One can define $\mathbb{G}$-equivariant intersection cohomology $I H_{\dot{G}}(Y):=H_{\mathbb{G}}(Y, I C(Y))$, which is also known to be a finite rank free graded $H_{\mathbb{G}}(p t)$-module, cf. [GKM]. Using this, it is straightforward to extend the proof of Proposisition 2.2.1 to an equivariant setting. Thus, one obtains the following result

Corollary 2.3.1. Let $Y=\bigsqcup_{s \in \mathcal{S}} Y_{s}$ be a stratification of a projective $\mathbb{G}$-variety $Y$ such that the strata $Y_{s}$ are $\mathbb{G}$-stable and satisfy the conditions of Proposition 2.2.1. Then, the natural map $H_{\mathbb{G}}(Y) \rightarrow I H_{\mathbb{G}}(Y)$ is a split imbedding of finite rank free $H_{\mathbb{G}}(p t)$-modules.

The space $H_{\mathbb{G}}(Y, \mathcal{L})$ may be viewed as a graded $\mathbb{C}[\text { Lie } \mathbb{G}]^{\text {Ad } \mathbb{G}}$-module, via the standard graded algebra isomorphism $H_{\mathbb{G}}^{\cdot}(p t)=\mathbb{C}[\text { Lie } \mathbb{G}]^{\text {Ad } \mathbb{G}}$. For any semisimple element $t \in$ Lie $\mathbb{G}$, let $\mathfrak{m}_{t} \subset \mathbb{C}[\text { Lie } \mathbb{G}]^{\text {Ad } \mathbb{G}}$ denote the maximal ideal corresponding to $\operatorname{Ad} \mathbb{G}(t)$, a closed conjugacy class in Lie $\mathbb{G}$. The quotient $H_{t}(Y, \mathcal{L}):=H_{\mathbb{G}}(Y, \mathcal{L}) / \mathfrak{m}_{t} \cdot H_{\mathbb{G}}(Y, \mathcal{L})$ will be referred to as a specialized equivariant cohomology group of $\mathcal{L}$. The grading on $H_{\mathbb{G}}(Y, \mathcal{L})$ gives rise to a canonical ascending filtration $E . H_{t}(Y, \mathcal{L})$, on $H_{t}(Y, \mathcal{L})$.

The augmentation ideal $\mathfrak{m}_{o} \subset \mathbb{C}[\text { Lie } \mathbb{G}]^{\text {Ad } \mathbb{G}}$ is a graded ideal. Hence, for $t=o \in \operatorname{Lie} \mathbb{G}$, the origin, the grading on $H_{\mathfrak{G}}(Y, \mathcal{L})$ descends to a well defined grading on $H_{o}(Y, \mathcal{L})$. In the case where $\mathcal{L}$ is, in addition, a semisimple $\mathbb{G}$-equivariant perverse sheaf of geometric origin, in the sense of [BBD], one has a natural graded space isomorphism with the nonequivariant cohomology of $\mathcal{L}$, cf. [G1],

$$
\operatorname{gr}^{E} H_{t}(Y, \mathcal{L}) \cong H_{o}^{\bullet}(Y, \mathcal{L}) \cong H^{\bullet}(Y, \mathcal{L}), \quad t \in \text { Lie } \mathbb{G} .
$$

A similar result also holds for the constant sheaf $\mathcal{L}=\mathbb{C}_{Y}$, provided the spectral sequence for the equivariant cohomology of $Y$ collapses, see eg. GKM. This is the case, for instance, if the odd cohomology of $Y$ vanishes. The latter condition is known to hold for any spherical Schubert variety $Y=\overline{\mathrm{Gr}}_{\lambda}$. 
Next, let $\mathbb{T}$ be a complex torus. Fix a smooth complex projective $\mathbb{T}$-variety $X$ and a subgroup $\mathbb{C}^{\times} \subset \mathbb{T}$. Assume that the $\mathbb{C}^{\times}$-action $t: x \mapsto t \cdot x$, on $X$, has a finite fixed point set $\mathcal{S}=X^{\mathbb{C}^{\times}}$. For each fixed point $s \in \mathcal{S}$, define the corresponding attracting set,

$$
X_{s}=\left\{x \in X \quad \mid \lim _{t \rightarrow 0} t \cdot x=s\right\}, \quad t \in \mathbb{C}^{\times} .
$$

One has the Bialynicki-Birula cell decomposition $X=\sqcup_{s \in \mathcal{S}} X_{s}$. Let $\bar{X}_{s}$ be the closure of $X_{s}$. For any $s \in \mathcal{S}$, the cell $X_{s}$ is isomorphic to a $\mathbb{C}$-vector space, see [BB], moreover, the complex $I C\left(\bar{X}_{s}\right)$ is known to be pointwise pure, cf. eg. [KL] or [G2].

Thus, Corollary 2.3.1 yields

Corollary 2.3.3. Assume that the Bialynicki-Birula decomposition $X=\sqcup_{s \in \mathcal{S}} X_{s}$ is a stratification of $X$, and let $s \in \mathcal{S}$. Then, the map $\kappa_{*}: H^{\bullet}\left(\bar{X}_{s}\right) \rightarrow I H^{\bullet}\left(\bar{X}_{s}\right)$, resp. the map $\kappa_{t}: H_{t}\left(\bar{X}_{s}\right) \rightarrow I H_{t}\left(\bar{X}_{s}\right)$, is injective for any $t \in \operatorname{Lie} \mathbb{T}$.

\section{Proofs: application of the Satake equivalence}

3.1 Write $E^{*}$ for the dual of a vector space $E$, and recall from 1.1 the $\mathfrak{s l}_{2}$-triple $e, h, f \in \mathfrak{g}$. Let $\rho \in \mathfrak{h}^{*}$ be the half-sum of positive roots. We will identify elements of $\mathfrak{h}^{*}$ with linear functions on $\mathfrak{g}$ via the standard imbedding $\mathfrak{h}^{*} \hookrightarrow \mathfrak{g}^{*}$.

Given $\lambda \in \mathfrak{g}^{*}$, write $\mathfrak{g}^{\lambda} \subset \mathfrak{g}$ for the isotropy Lie algebra of the ad* $\mathfrak{g}$-action on $\mathfrak{g}^{*}$ (the identification $\mathfrak{g} \simeq \mathfrak{g}^{*}$ provided by an invariant form, makes $\mathfrak{g}^{\lambda}$ the centralizer of $\lambda$ ). We set $\eta:=\operatorname{ad}^{*} e(\rho)$. It is easy to check that one has $\mathfrak{g}^{\eta}=\mathfrak{g}^{e}$, resp. $\mathfrak{g}^{\eta+\rho}=\mathfrak{g}^{e+h}$. According to Kostant [Ko3], for any $\mu \in \mathfrak{h}^{*}$, the Lie algebra $\mathfrak{g}^{\eta+\mu}$ is abelian and one has $\operatorname{dim} \mathfrak{g}^{\eta+\mu}=$ rk $\mathfrak{g}$.

Let $\operatorname{Rep} \mathfrak{g}$ be the tensor category of finite dimensional representations of the Lie algebra $\mathfrak{g}$. Let $h$ be the semisimple element from the $\mathfrak{s l}_{2}$-triple, and let $V \in \operatorname{Rep} \mathfrak{g}$. The eigen-space decomposition with respect to the $h$-action in $V$ gives a grading $V \cdot=\bigoplus_{m \in \mathbb{Z}} V_{m}$, to be referred to as the Brylinski grading. We also define an increasing filtration $F . V$, called the Brylinski filtration, defined by $F_{k} V:=\bigoplus_{m \leq k} V_{m}$.

In the special case where $V=\mathfrak{g}$, the adjoint representation, the Brylinski filtration restricts to a filtration on any Lie subalgebra $\mathfrak{k} \subset \mathfrak{g}$. This makes the enveloping algebra Uk a filtered algebra with respect to the induced filtration (not to be confused with the standard filtration on an enveloping algebra).

Write Vect (resp. Vect.), for the tensor category of finite dimensional filtered resp. graded, vector spaces. Assigning to a $\mathfrak{g}$-representation the underlying vector space equipped with the Brylinski filtration, resp. Brylinski grading, yields a fiber functor $F: \operatorname{Rep} \mathfrak{g} \rightarrow$ Vect, resp. Rep $\mathfrak{g} \rightarrow$ Vect. .

3.2 We have the Langlands dual data $\left({ }^{L} G,{ }^{L} T\right)$, see $₫ 1.2$, and we put $\mathfrak{t}=\operatorname{Lie}^{L_{T}}$. Thus, there is a diagram of canonical morphisms of affine algebraic varieties

$$
\eta+\mathfrak{h}^{*} \stackrel{\eta+\mu \mapsto W(\mu)}{\longrightarrow} \mathfrak{h}^{*} / W \simeq \mathfrak{t} / W \simeq \operatorname{Spec}\left(\mathbb{C}\left[\operatorname{Lie}^{L^{L}} G\right]^{\operatorname{Ad}^{L} G}\right)
$$

where the last isomorphism is the Chevalley isomorphism. 
We may view ${ }^{L} G$ as a subgroup of ${ }^{L} G[[z]]$ formed by constant loops. The loop Grassmannian thus becomes a topological ${ }^{L} G$-space with respect to the left ${ }^{L} G$-action. Therefore, associated with any ${ }^{L} G$-equivariant complex $\mathcal{L}$, on $\mathrm{Gr}$, and an element $\mu \in \mathfrak{h}^{*}=\mathfrak{t}$, there is a filtered vector space $H_{\mu}(\mathrm{Gr}, \mathcal{L})$, a specialized ${ }^{L} G$-equivariant cohomology group, see 22.3 . Equivalently, the same group may be obtained by specializing $H_{L_{T}}(\mathrm{Gr}, \mathcal{L}) \cong$ $\mathbb{C}[\mathfrak{t}] \bigotimes_{\mathbb{C}[\mathfrak{t}] W} H_{\dot{L}_{G}}(\mathrm{Gr}, \mathcal{L})$, the ${ }^{L} T$-equivariant cohomology, at the point $\mu \in \mathfrak{t}$.

In [G1, Proposition 1.7.2], we have proved, cf. also [BFM],

Proposition 3.2.2. For any $\mu \in \mathfrak{h}^{*}$, there is a canonical filtered algebra isomorphism

$$
\varphi_{\mu}: H_{\mu}(\mathrm{Gr}) \stackrel{\sim}{\rightarrow} \mathrm{Ug}^{\eta+\mu} .
$$

For $\mu=o$ (the origin), this isomorphism respects the canonical gradings on both sides.

Remark 3.2.4. For $\mu=o$, we have $H_{o}^{\bullet}(\mathrm{Gr})=H^{\bullet}(\mathrm{Gr})$, by (2.3.2), and (3.2.3) reduces to the graded isomorphism mentioned in Remark 1.3.4(ii).

3.3 Let $\mathcal{P}(\mathrm{Gr})$ be the tensor category of ${ }^{L} G[[z]]$-equivariant perverse sheaves on $\mathrm{Gr}$ with compact support, and equipped with a monoidal structure given by convolution. The category $\mathcal{P}(\mathrm{Gr})$ is known to be semisimple, cf. eg. [MV]. Taking specialized equivariant cohomology gives a family fiber functors parametrized by the elements $\mu \in \mathfrak{h}^{*}$,

$$
H_{\mu}(\mathrm{Gr},-): \mathcal{P}(\mathrm{Gr}) \rightarrow \text { Vect } \quad\left(\text { resp. } H_{o}(\mathrm{Gr},-): \mathcal{P}(\mathrm{Gr}) \rightarrow \text { Vect. }\right), \quad \mathcal{L} \mapsto H_{\mu}(\mathrm{Gr}, \mathcal{L}) .
$$

Using the isomorphism in (2.3.2), we will often identify specialized and ordinary cohomology functors $H_{o}^{\bullet}(\mathrm{Gr},-)=H^{\bullet}(\mathrm{Gr},-)$.

View $\rho$ as an element of $\mathfrak{t}$ and observe that the $\rho$-fixed point set in $\mathrm{Gr}$ is equal to the set (1.2.1), of ${ }^{L} T$-fixed points. For each $\mu \in \mathfrak{h}_{\mathbb{Z}}^{*}$, let $i_{\mu}:\{\mu\} \hookrightarrow \mathrm{Gr}$ denote the corresponding one point imbedding. The Localization theorem in equivariant cohomology yields the following fixed point decomposition, see [G1, (3.6.1)],

$$
H_{\rho}(\mathrm{Gr}, \mathcal{L})=\oplus_{\mu \in \mathfrak{h}_{\mathbb{Z}}^{*}} H_{\rho}\left(i_{\mu} \mathcal{L}\right), \quad \forall \mathcal{L} \in \mathcal{P}(\mathrm{Gr}) .
$$

Theorem 3.3.2 (Geometric Satake equivalence). (i) There is a monoidal equivalence $\mathbf{S}: \mathcal{P}(\mathrm{Gr}) \stackrel{\sim}{\rightarrow} \operatorname{Rep} \mathfrak{g}$ and, for each $\mu \in \mathfrak{h}^{*}$, an isomorphism $\Phi_{\mu}: H_{\mu}(\mathrm{Gr},-) \stackrel{\sim}{\rightarrow} F \circ \mathbf{S}(-)$, of monoidal functors, such that

- For any dominant weight $\lambda$, one has an isomorphism $\mathbf{S}\left(\operatorname{IC}\left(\overline{\mathrm{Gr}}_{\lambda}\right)\right) \cong V_{\lambda}$.

- For any $\mathcal{L} \in \mathcal{P}(\mathrm{Gr})$ and $u \in H_{\mu}(\mathrm{Gr})$, the following diagram commutes:

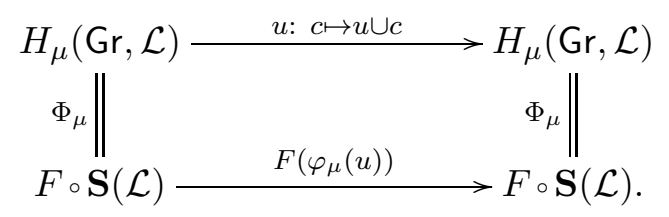

- The isomorphism $\Phi_{\mu}$ sends the canonical filtration on the specialized equivariant cohomology to the Brylinski filtration.

(ii) For $\mu=\rho$, the isomorphism $\Phi_{\rho}$ sends the fixed point decomposition (3.3.1) to the weight decomposition with respect to the Cartan subalgebra $\mathfrak{g}^{\eta+\rho}$. 
(iii) For $\mu=o$, the natural cohomology grading on $H_{o}^{\cdot}(\mathrm{Gr},-)$ goes, under the isomorphism $\Phi_{o}$, to the Brylinski grading.

This theorem was first proved in [G1, (see Theorem 1.4.1 and Theorem 1.7.6 in G1]), using some earlier results by Lusztig $[\mathrm{Lu}$, and a crucial idea due to Drinfeld. Exploiting some additional unpublished ideas of Drinfeld concerning the commutativity constraint in $\mathcal{P}(\mathrm{Gr})$, an alternate, more elegant proof of the monoidal equivalence of the theorem was obtained later by Mirković and Vilonen [MV].

3.4 Recall the notation from (1.3.1) and (2.1.1).

Lemma 3.4.1. For any $\mu \in \mathfrak{h}^{*}$, and an anti-dominant weight $\lambda \in \mathfrak{h}_{\mathbb{Z}}^{*}$, there is a filtered algebra isomorphism

$$
H_{\mu}\left(\overline{\mathrm{Gr}}_{\lambda}\right) \cong \mathrm{Ug}^{\eta+\mu} / \operatorname{Ann}\left[\mathfrak{g}^{\eta+\mu} ; v_{\lambda}\right]
$$

Furthermore, in the special case $\mu=o$, the above isomorphism respects the gradings.

Proof. We write Ann $\left[-; \kappa_{*}(1)\right]$ for an annihilator of the element $\kappa_{*}(1) \in I H_{\mu}\left(\overline{\mathrm{Gr}}_{\lambda}\right)$.

One has a natural restriction map res ${ }_{\lambda}: H_{\mu}(\mathrm{Gr}) \rightarrow H_{\mu}\left(\overline{\mathrm{Gr}}_{\lambda}\right)$. This map is surjective since the spaces $\overline{\mathrm{Gr}}_{\lambda}$ and $\mathrm{Gr}$ have compatible cell decompositions by Iwahori-orbits (which have even real dimension). By Corollary 2.3 .3 applied to the variety $X=\overline{\mathrm{Gr}}_{\lambda}$, we have

$$
\operatorname{res}_{\lambda}\left(\operatorname{Ann}\left[H_{\mu}(\mathrm{Gr}) ; \kappa_{*}(1)\right]\right) \subset \operatorname{Ann}\left[H_{\mu}\left(\overline{\mathrm{Gr}}_{\lambda}\right) ; \kappa_{*}(1)\right]=0 .
$$

This yields an inclusion $\operatorname{Ann}\left[H_{\mu}(\mathrm{Gr}) ; \kappa_{*}(1)\right] \subset \operatorname{Ker}\left(\mathrm{res}_{\lambda}\right)$. Clearly, the $H_{\mu}(\mathrm{Gr})$-action on $I H_{\mu}\left(\overline{\mathrm{Gr}}_{\lambda}\right)$ factors through the restriction $H_{\mu}(\mathrm{Gr}) \rightarrow H_{\mu}\left(\overline{\mathrm{Gr}}_{\lambda}\right)$. Hence, we must have $\operatorname{Ann}\left[H_{\mu}(\mathrm{Gr}) ; \kappa_{*}(1)\right]=\operatorname{Ker}\left(\operatorname{res}_{\lambda}\right)$. The map res ${ }_{\lambda}$ therefore induces an isomorphism

$$
H_{\mu}(\mathrm{Gr}) / \operatorname{Ann}\left[H_{\mu}(\mathrm{Gr}) ; \kappa_{*}(1)\right] \stackrel{\sim}{\rightarrow} H_{\mu}\left(\overline{\mathrm{Gr}}_{\lambda}\right) .
$$

On the other hand, the isomorphism $\varphi_{\mu}: H_{\mu}(\mathrm{Gr}) \stackrel{\sim}{\rightarrow} \mathrm{Ug}^{\eta+\mu}$ in (3.2.3) provides, by Corollary 3.3 .2 , the following bijections

$$
\begin{aligned}
& \operatorname{Ann}\left[H_{\mu}(\mathrm{Gr}) ; \kappa_{*}(1)\right] \stackrel{\sim}{\rightarrow} \operatorname{Ann}\left[\mathfrak{g}^{\eta+\mu} ; v_{\lambda}\right], \quad \text { resp. } \\
& \operatorname{Ann}\left[H_{\mu}(\mathrm{Gr}) ; I H_{\mu}\left(\overline{\mathrm{Gr}}_{\lambda}\right)\right] \stackrel{\sim}{\rightarrow} \operatorname{Ann}\left[\mathfrak{g}^{\eta+\mu} ; V_{\lambda}\right] .
\end{aligned}
$$

The isomorphism of the lemma now follows from (3.4.3).

Proof of Theorem 1.3.2. The first isomorphism in (1.3.3) is the statement of Lemma 3.4.1 for $\mu=o$. To prove the second isomorphism in (1.3.3), we take $\mu=\rho$. By Proposition 3.2.2, we get $H_{\rho}(\mathrm{Gr})=\mathrm{Ug}^{\eta+\rho}=\mathrm{Ug}^{e+h} \cong \mathrm{Ug}^{h}=\mathrm{Uh}$, where the third isomorphism is given by conjugation by $u:=\exp e$, a unipotent element. It is straightforward to verify that such a conjugation sends the Brylinski filtration on $\mathfrak{g}^{\eta+\rho}$ to the filtration on $\mathfrak{h}$ that was defined in 1.3. Further, it is clear from Theorem 3.3.2(ii) that the ideal $\operatorname{Ann}\left[U\left(g^{\eta+\mu}\right) ; V_{\lambda}\right] \subset \mathrm{Ug}^{\eta+\rho}$ goes, via conjugation by $u$, to the ideal $\operatorname{Ann}\left[\mathfrak{h} ; V_{\lambda}\right]=I\left(V_{\lambda}\right) \subset$ Uh. Thus, we obtain filtered algebra isomorphisms

$$
H_{\rho}\left(\overline{\operatorname{Gr}}_{\lambda}\right) \stackrel{(3.4 .2)}{=} \mathrm{Ug} \mathfrak{g}^{\eta+\rho} / \operatorname{Ann}\left[\mathfrak{g}^{\eta+\rho} ; v_{\lambda}\right] \stackrel{\operatorname{Ad} u}{=} \mathrm{Uh} / I\left(V_{\lambda}\right)=\mathbb{C}\left[\operatorname{Spec} V_{\lambda}\right] .
$$


Now, we combine the above isomorphisms for $\mu=o$ and for $\mu=\rho$. In this way, we obtain a chain of graded algebra isomorphisms

$$
\mathrm{Ug} \mathfrak{g}^{e} / \operatorname{Ann}\left[\mathfrak{g}^{e} ; v_{\lambda}\right]=\mathrm{Ug}^{\eta} / \operatorname{Ann}\left[\mathfrak{g}^{\eta} ; v_{\lambda}\right] \cong H^{\bullet}\left(\overline{\mathrm{Gr}}_{\lambda}\right) \cong \operatorname{gr} . H_{\rho}\left(\overline{\mathrm{Gr}}_{\lambda}\right)=\operatorname{gr} . \mathbb{C}\left[\operatorname{Spec} V_{\lambda}\right]
$$

Thus, the isomorphism on the right of (1.3.3) is provided by the composite.

Proof of Proposition 1.5.3. We use Proposition 3.2 .2 and Theorem 3.3.2 in the case $\mu=o$, cf. Remark 3.2.4. Thus, we may define the required map $f_{o}$ as a composite of the following chain of maps, cf. (1.5.1),

$$
\begin{aligned}
V_{\lambda}=I H^{\bullet}\left(\overline{\mathrm{Gr}}_{\lambda}\right)=H^{\bullet}\left(\overline{\mathrm{Gr}}_{\lambda}, I C\left(\overline{\mathrm{Gr}}_{\lambda}\right)\right) & \longrightarrow H^{\bullet}\left(\mathrm{Gr}_{\lambda}, \jmath_{\lambda}^{*} I C\left(\overline{\mathrm{Gr}}_{\lambda}\right)\right)=H^{\bullet}\left(\mathrm{Gr}_{\lambda}, \mathbb{C}_{\mathrm{Gr}_{\lambda}}\right) \\
& =H^{\bullet}\left(\mathrm{Gr}_{\lambda}\right)=H^{\bullet}\left({ }^{L} G / P_{\lambda}\right)=\mathrm{Sh}^{W_{\lambda}} / \mathfrak{m}_{o} \cdot \mathrm{Sh}^{W_{\lambda}} .
\end{aligned}
$$

It remains to prove the surjectivity of the composite map (1.5.2). According to the above, this composite may be identified with the restriction map $H^{\bullet}\left(\overline{\mathrm{Gr}}_{\lambda}\right) \rightarrow H^{\bullet}\left(\mathrm{Gr}_{\lambda}\right)$. The latter map is surjective since the spaces in question have compatible partitions into cells of even real dimension.

3.5 Proof of Theorem 1.6.1. Part (i) is clear from the proof of Proposition 1.5.3 since, for a minuscule weight $\mu$, each of the imbeddings in (1.5.1) is a bijection.

To prove (ii), for any anti-dominant weigth $\lambda$, write $I C_{\lambda}:=I C\left(\overline{\mathrm{Gr}}_{\lambda}\right)$. Let $D^{b}\left(\mathrm{Gr},{ }^{L_{T}}\right)$ be the bounded ${ }^{L} T$-equivariant derived category of constructible complexes on $\mathrm{Gr}$, with compact support, as defined in [BL1]. For any objects $\mathcal{L}, \mathcal{L}^{\prime} \in D^{b}\left(\mathrm{Gr},{ }^{L} T\right)$, we define a family of specialized equivariant Ext-groups

$$
\operatorname{Ext}_{\lambda}\left(\mathcal{L}, \mathcal{L}^{\prime}\right):=\operatorname{Ext}_{D^{b}\left(\mathrm{Gr}, L_{T}\right)}\left(\mathcal{L}, \mathcal{L}^{\prime}\right) / \mathfrak{m}_{\lambda} \cdot \operatorname{Ext}_{D^{b}(\mathrm{Gr}, L T)}^{\bullet}\left(\mathcal{L}, \mathcal{L}^{\prime}\right), \quad \lambda \in \mathfrak{h}^{*}
$$

Let $\mu$ be a minuscule anti-dominant weight, and let $j_{\mu}: \mathrm{Gr}_{\mu}=\overline{\mathrm{Gr}}_{\mu} \hookrightarrow \mathrm{Gr}$ be the corresponding closed imbedding. Clearly, we have $I C_{\mu}=\left(j_{\mu}\right) ! \mathbb{C}_{\mathrm{Gr}_{\mu}}$. Further, given $\lambda \in \mathfrak{h}^{*}$, put $J(\lambda):=\operatorname{Ann}\left[\mathfrak{g}^{\eta+\lambda} ; V_{\mu}\right]$. From Lemma 3.4.1 and the proof of Theorem 1.3.2, we deduce an isomorphism $V_{\mu}=\mathrm{Ug}^{\eta+\lambda} / J(\lambda)$.

Let $\mathcal{M} \in \mathcal{P}(\mathrm{Gr})$ be a perverse sheaf supported on the connected component of the loop Grassmannian that contains the orbit $\mathrm{Gr}_{\mu}$, and let $V:=\mathbf{S}(\mathcal{M}) \in \operatorname{Rep} \mathfrak{g}$ be the representation corresponding to $\mathcal{M}$ via the Satake equivalence. By Theorem 1.10.3 of G1] (whose proof depends on G2] in an essential way), for any $\lambda \in \mathfrak{h}^{*}$ there are canonical vector space isomorphisms

$$
\begin{aligned}
H_{\lambda}\left(j_{\mu}^{!} \mathcal{M}\right)=\operatorname{Ext}_{\lambda}\left(\left(j_{\mu}\right) ! \mathbb{C}_{\mathrm{Gr}_{\mu}}, \mathcal{M}\right) & =\operatorname{Ext}_{\lambda}\left(I C_{\mu}, \mathcal{M}\right) \frac{\text { G1], Thm. 1.10 }}{} \operatorname{Hom}_{\mathfrak{g}^{\eta+\lambda}}\left(V_{\mu}, V\right) \\
& =\operatorname{Hom}_{\mathfrak{g}^{\eta+\lambda}}\left(\mathrm{Ug}^{\eta+\lambda} / J(\lambda), V\right)=V^{J(\lambda)}
\end{aligned}
$$

For $\lambda=0$, we have $\mathfrak{g}^{\eta}=\mathfrak{g}^{e}$, in particular, $\operatorname{Ann}\left[\mathfrak{g}^{e} ; v_{\mu}\right]=J(0)$. In this case, all the isomorphisms in (3.5.1) respect the gradings. On the other hand, we may take $\lambda=\rho$. Then, according to (3.4.4), we get

$$
V_{\mu}=H_{\rho}\left(\mathrm{Gr}, I C_{\mu}\right)=H_{\rho}\left(\mathrm{Gr}_{\mu}\right)=\mathrm{Uh} / I\left(\operatorname{Spec} V_{\mu}\right) .
$$


Hence, the ideal $J(\rho)$ is obtained from $I\left(\operatorname{Spec} V_{\mu}\right)$ via the adjoint action by the element $u$, cf. (3.4.4). Thus, we obtain a graded, resp. filtered, isomorphism

$$
H^{\bullet}\left(j_{\mu}^{!} \mathcal{M}\right) \cong V^{\operatorname{Ann}\left[\mathfrak{g}^{e} ; v_{\mu}\right]}, \quad \text { resp. } \quad H_{\rho}\left(j_{\mu}^{!} \mathcal{M}\right) \cong V^{I\left(\operatorname{Spec} V_{\mu}\right)}
$$

Observe that the complex $j_{\mu}^{!} \mathcal{M}$ is pure, by pointwise purity of any object of category $\mathcal{P}(\mathrm{Gr})$. Hence, the isomorphism in (2.3.2) applies to the complex $\mathcal{L}=j_{\mu}^{!} \mathcal{M}$, and we deduce that $H^{\bullet}\left(j_{\mu}^{!} \mathcal{M}\right) \cong \operatorname{gr} H_{\rho}\left(j_{\mu}^{!} \mathcal{M}\right)$. Further, the fixed point decomposition (3.3.1) shows that $V^{I\left(\operatorname{Spec} V_{\mu}\right)}=\oplus_{\nu \in W(\mu)} V(\nu)$. Hence, we compute $\operatorname{dim} V^{I\left(\operatorname{Spec} V_{\mu}\right)}=\operatorname{dim} V(\mu) \cdot|W(\mu)|$, since $\operatorname{dim} V(\nu)=\operatorname{dim} V(\mu)$ for any $\nu \in W(\mu)$. The proof is completed by equating dimensions of the various isomorphic vector spaces considered above,

$$
\begin{aligned}
\operatorname{dim} V^{\operatorname{Ann}\left[\mathfrak{g}^{e} ; v_{\mu}\right]}=\operatorname{dim} H^{\bullet}\left(j_{\mu}^{!} \mathcal{M}\right) & =\operatorname{dimgr} H_{\rho}\left(j_{\mu}^{!} \mathcal{M}\right)=H_{\rho}\left(j_{\mu}^{!} \mathcal{M}\right) \\
& =\operatorname{dim} V^{I\left(\operatorname{Spec} V_{\mu}\right)}=\operatorname{dim} V(\mu) \cdot\left|W / W_{\mu}\right| .
\end{aligned}
$$

\section{Kostant's theorem on $\mathrm{Ad} G$-invariant polynomials.}

4.1 Let $G$ be the adjoint group of a semisimple Lie algebra $\mathfrak{g}$. The group $G$ acts on $\mathfrak{g}^{*}$ by the (co)adjoint action. In this section, we discuss various incarnations of the famous result, due to Kostant [Ko1], concerning the structure of the coadjoint quotient $\operatorname{map} \varpi: \mathfrak{g}^{*} \rightarrow \mathfrak{g}^{*} / / \operatorname{Ad} G$.

The most essential part of Kostant's result reads

Theorem 4.1.1 (Kostant). The algebra $\mathrm{Sg}$ is a free graded $\mathrm{Sg}^{G}$-module; furthermore, the quotient algebra $\mathrm{Sg} / \mathfrak{m} \cdot \mathrm{Sg}$ is a normal domain, for any maximal ideal $\mathfrak{m} \subset \mathrm{Sg}^{G}$.

A key geometric result in the original approach of Ko1 was the normality of the nilpotent variety in $\mathfrak{g}$. Kostant's proof of that result involved certain properties of CohenMacauley rings which boil down, essentially, to the Serre normality criterion, see [Se]. A simplified argument, that bypasses references to Serre's criterion, was later found by Bernstein and Lunts [BL2, cf. also [CG], $\S 6.7$ for an exposition. Below, we discuss an alternate approach to Theorem 4.1.1 which is of a more 'topological' nature.

To explain our approach, it will be convenient to reformulate Kostant's result. To this end, let $\mathcal{B}$ be the flag variety for $G$, viewed as the variety of Borel subalgebras in $\mathfrak{g}$. One introduces an incidence variety $\widetilde{\mathfrak{g}}^{*}:=\left\{(x, \mathfrak{b}) \in \mathfrak{g}^{*} \times \mathcal{B}|x|_{[\mathfrak{b}, \mathfrak{b}]}=0\right\}$ that fits into the following commutative diagram,

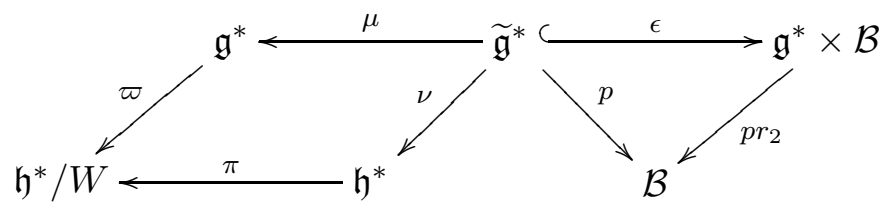

In this diagram, $\pi$ is a finite flat morphism, since $\mathbb{C}[\mathfrak{h}]$ is free over $\mathbb{C}[\mathfrak{h}]^{W}$, and the map $\nu:\left.x \mapsto x\right|_{\mathfrak{b} /[\mathfrak{b}, \mathfrak{b}]}$ is a smooth morphism induced by restriction via the diagram $\mathfrak{g} \hookleftarrow \mathfrak{b} \rightarrow \mathfrak{b} /[\mathfrak{b}, \mathfrak{b}]=: \mathfrak{h}, \forall \mathfrak{b} \in \mathcal{B}$, cf. eg. [CG], formula (7.3.1). Further, the map $\mu$, resp. $p=p r_{2} \circ \epsilon$, is induced by the first, resp. second, projection of $\mathfrak{g} \times \mathcal{B}$. 
The commutative parallelogram on the left of (4.1.2) is known as the GrothendieckSpringer resolution, cf. eg. [CG, §3.2]. This parallelogram is not a cartesian square; in fact, the map $\mu \times \nu$ may be factored as follows

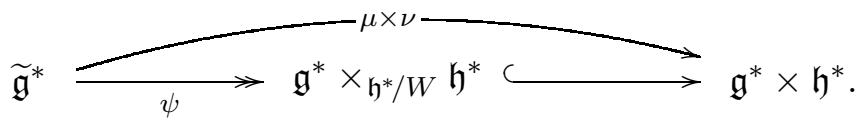

Here, the map $\psi$ is dominant and proper, hence, surjective. Further, by definition, we have $\mathbb{C}\left[\mathfrak{g}^{*} \times \mathfrak{h}^{*} / W \mathfrak{h}^{*}\right]=\mathrm{Sg} \otimes_{\mathrm{Sh}^{W}} \mathrm{Sh}$.

The following version of Kostant's result is often more convenient, cf. eg. [BBM].

Theorem 4.1.4. The morphism (4.1.3) induces an algebra isomorphism

$$
\psi^{*}: \mathrm{Sg} \otimes_{\mathrm{Sh}^{W}} \mathrm{Sh} \stackrel{\sim}{\rightarrow} \Gamma\left(\widetilde{\mathfrak{g}}^{*}, \mathcal{O}_{\widetilde{\mathfrak{g}}^{*}}\right) .
$$

Furthermore, we have $H^{i}\left(\widetilde{\mathfrak{g}}^{*}, \mathcal{O}_{\widetilde{\mathfrak{g}}^{*}}\right)=0$, for any $i>0$.

Theorem 4.1.4 implies Theorem 4.1.1. First of all, we recall that any nonnegatively graded flat $\mathrm{Sg}^{G}$-module is necessarily free. Therefore, to prove the first statement of Theorem 4.1.1 it suffices to show that $\mathrm{Sg}$ is a flat $\mathrm{Sg}^{G}$-module. But this follows from Theorem 4.1.4 by a flat base change. In fact, the classical result says that $\mathrm{Sh}$ is a free $\mathrm{Sh}^{W}$-module of rank $|W|$. Hence, $\mathrm{Sg}$ is flat over $\mathrm{Sg}^{G}$ if and only if $\mathrm{Sg} \otimes_{\mathrm{Sh}^{W}} \mathrm{Sh}$ is flat over $\mathrm{Sh}$, by the base change. Applying Theorem 4.1.4, we see that it suffices to prove that $\Gamma\left(\widetilde{\mathfrak{g}}^{*}, \mathcal{O}_{\widetilde{\mathfrak{g}}^{*}}\right)$ is flat over $\mathrm{Sh}$. But this is clear since $\nu$ is a smooth morphism, hence, it is flat.

The second statement of Theorem 4.1.1 is proved similarly by restricting diagram (4.1.2) to a fiber of the map $\nu$, which is a smooth morphism. Specifically, given a maximal ideal $\mathfrak{m} \subset \mathrm{Sg}^{G}=\mathrm{Sh}^{W}$, choose a maximal ideal of the algebra $\mathrm{Sh}=\mathbb{C}\left[\mathfrak{h}^{*}\right]$ over $\mathfrak{m}$, i.e. an element $\chi \in \mathfrak{h}^{*}$ such that, for the maximal ideal $(\chi) \subset \mathbb{C}\left[\mathfrak{h}^{*}\right]$ associated with $\chi$, one has $(\chi) \cap \mathrm{Sh}^{W}=\mathfrak{m}$. We set $\mathbb{C}_{\chi}=\mathrm{Sh} /(\chi)$, resp. $\mathbb{C}_{\mathfrak{m}}=\mathrm{Sh}^{W} / \mathfrak{m}$. Also, let $\widetilde{\mathfrak{g}}_{\chi}^{*}:=\nu^{-1}(\chi)$ be the fiber of the map $\nu$ over the point $\chi \in \mathfrak{h}^{*}$.

Then, from diagram (4.1.2), we get $\Gamma\left(\widetilde{\mathfrak{g}}_{\chi}^{*}, \mathcal{O}_{\widetilde{\mathfrak{g}}_{\chi}^{*}}\right)=\Gamma\left(\widetilde{\mathfrak{g}}^{*}, \mathcal{O}_{\widetilde{\mathfrak{g}}^{*}}\right) \otimes_{\mathrm{Sh}} \mathbb{C}_{\chi}$. Hence, using Theorem 4.1.4 we find

$$
\Gamma\left(\widetilde{\mathfrak{g}}_{\chi}^{*}, \mathcal{O}_{\widetilde{\mathfrak{g}}_{\chi}^{*}}\right)=\left(\mathrm{Sg} \otimes_{\mathrm{Sh}} W \mathrm{Sh}\right) \otimes_{\mathrm{Sh}} \mathbb{C}_{\chi}=\mathrm{Sg} \otimes_{\mathrm{Sh}^{W}} \mathbb{C}_{\mathfrak{m}}=\mathrm{Sg} / \mathfrak{m} \cdot \mathrm{Sg}
$$

The scheme $\widetilde{\mathfrak{g}}_{\chi}^{*}$ being a smooth connected variety, we conclude that the algebra $\Gamma\left(\widetilde{\mathfrak{g}}_{\chi}^{*}, \mathcal{O}_{\widetilde{\mathfrak{g}}_{\chi}^{*}}\right)$ is a normal domain, and we are done.

4.2 A 'topological' proof of Theorem 4.1.4. Given a coherent sheaf $\mathcal{V}$, we write $\mathcal{S} y m \mathcal{V}$ for the corresponding symmetric algebra, a quasi-coherent sheaf.

Write $\mathfrak{g}_{\mathcal{B}}:=\mathfrak{g} \otimes \mathcal{O}_{\mathcal{B}}$ for the trivial sheaf on $\mathcal{B}$ with fiber $\mathfrak{g}$, and $\mathfrak{n}_{\mathcal{B}} \subset \mathfrak{g}_{\mathcal{B}}$ for a (nontrivial) subsheaf whose fiber at each point $\mathfrak{b} \in \mathcal{B}$ is $\mathfrak{n}=[\mathfrak{b}, \mathfrak{b}]$, the nilradical of the Borel subagebra $\mathfrak{b}$. There is a canonical isomorphism

$$
\mathfrak{n}_{\mathcal{B}} \cong T_{\mathcal{B}}^{*}:=\text { cotangent sheaf on } \mathcal{B} \text {. }
$$

The closed imbedding $\epsilon$, in diagram (4.1.2), makes $p: \widetilde{\mathfrak{g}}^{*} \rightarrow \mathcal{B}$ a sub-vector bundle of the trivial bundle $p r_{2}: \mathfrak{g}^{*} \times \mathcal{B} \rightarrow \mathcal{B}$. It is clear that the sheaf of sections of the vector 
bundle dual to $\widetilde{\mathfrak{g}}^{*}$ is isomorphic naturally to $\mathfrak{g}_{\mathcal{B}} / \mathfrak{n}_{\mathcal{B}}$, a quotient sheaf. The vector bundle projection $p$ being affine, we get $H^{i}\left(\widetilde{\mathfrak{g}}^{*}, \mathcal{O}_{\widetilde{\mathfrak{g}}^{*}}\right)=H^{i}\left(\mathcal{B}, p . \mathcal{O}_{\tilde{\mathfrak{g}}^{*}}\right)=H^{i}\left(\mathcal{B}, \operatorname{Sym}\left(\mathfrak{g}_{\mathcal{B}} / \mathfrak{n}_{\mathcal{B}}\right)\right)$.

The computation of the rightmost sheaf cohomology group given below is based on an old idea that goes back to Jantzen and Miličič.

For each $q=0,1, \ldots$, let $\mathcal{K}^{q}:=\left(\mathcal{S}_{y m} \mathfrak{g}_{\mathcal{B}}\right) \bigotimes_{\mathcal{O}_{\mathcal{B}}}\left(\wedge^{q} \mathfrak{n}_{\mathcal{B}}\right)=\mathrm{Sg} \otimes \wedge^{q} \mathfrak{n}_{\mathcal{B}}$, a quasi-coherent sheaf on $\mathcal{B}$. The sheaf imbedding $\mathfrak{n}_{\mathcal{B}} \hookrightarrow \mathfrak{g}_{\mathcal{B}}$ gives, in a standard way, a Koszul differential $\mathcal{K}^{\bullet} \rightarrow \mathcal{K}^{\cdot-1}$. This differential is a sheaf morphism that makes $\mathcal{K}^{\bullet}$ a resolution of the sheaf $\operatorname{Sym}\left(\mathfrak{g}_{\mathcal{B}} / \mathfrak{n}_{\mathcal{B}}\right)$. Further, using (4.2.1), one can write $\wedge^{\bullet} \mathfrak{n}_{\mathcal{B}}=\wedge^{\bullet} T_{\mathcal{B}}^{*}=\Omega_{\mathcal{B}}^{*}$, the sheaf of algebraic differential forms on $\mathcal{B}$. Thus, we obtain $\mathcal{K}^{\bullet}=\mathrm{Sg} \otimes \Omega_{\mathcal{B}}$.

The resolution $\mathcal{K}^{\bullet} \rightarrow \mathcal{S}_{y m}\left(\mathfrak{g}_{\mathcal{B}} / \mathfrak{n}_{\mathcal{B}}\right) \rightarrow 0$ may be used for computing sheaf cohomology. Specifically, there is a convergent third quadrant spectral sequence

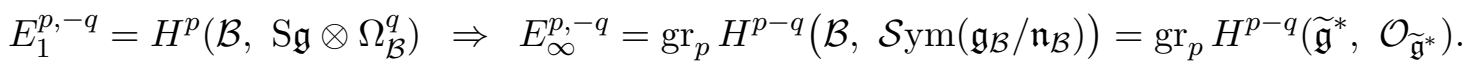

We have $H^{p}\left(\mathcal{B}, \mathrm{Sg} \otimes \Omega_{\mathcal{B}}^{q}\right)=\mathrm{Sg} \otimes H^{p}\left(\mathcal{B}, \Omega_{\mathcal{B}}^{q}\right)=\mathrm{Sg} \otimes H^{p, q}(\mathcal{B})$, by the Hodge decomposition for the de Rham cohomology of a Kähler manifold. Recall that, for the flag manifold $\mathcal{B}$, the group $H^{p, q}(\mathcal{B})$ vanishes unless $p=q$. We conclude that the spectral sequence degenerates at the $E_{1}$-term. Moreover, we deduce that the cohomology group gr $H^{i}\left(\widetilde{\mathfrak{g}}^{*}, \mathcal{O}_{\widetilde{\mathfrak{g}}^{*}}\right)$ vanishes for any $i>0$, and there are graded algebra isomorphisms

$$
\operatorname{gr} H^{0}\left(\widetilde{\mathfrak{g}}^{*}, \mathcal{O}_{\widetilde{\mathfrak{g}}^{*}}\right)=E_{\infty}=E_{1}=\mathrm{Sg} \otimes H^{\bullet}(\mathcal{B}) .
$$

Here, the grading on the leftmost term is the one that comes from the $\mathbb{C}^{\times}$-action on $\widetilde{\mathfrak{g}}^{*}$ by dilations along the fibers, and the grading on the tensor product on the right is the tensor product grading induced by the natural grading on a symmetric algebra and the grading on $H^{\bullet}(\mathcal{B})$ such that $H^{2 i}(\mathcal{B})$ is placed in degree $i$. Thus, we have proved that $H^{i}\left(\widetilde{\mathfrak{g}}^{*}, \mathcal{O}_{\widetilde{\mathfrak{g}}^{*}}\right)=0, \forall i>0$.

To complete the proof, we use the Borel isomorphism and write $\mathrm{Sh}$, a free graded $\mathrm{Sh}^{W}$-module, in the form $\mathrm{Sh}=\mathrm{Sh}^{W} \otimes H^{\bullet}(\mathcal{B})$, where the cohomology of the flag manifold is equipped with the same grading as above.

Further, view the map $\psi^{*}$, in the statement of the theorem, as a map of $\mathbb{C}\left[\mathfrak{g}^{*}\right]$-modules. The kernel of this map must be a torsion $\mathbb{C}\left[\mathfrak{g}^{*}\right]$-submodule since the morphism (4.1.3) is easily seen to be an isomorphism at the generic point. On the other hand, we have

$$
\mathrm{Sg} \otimes_{\mathrm{Sh}^{W}} \mathrm{Sh}=\mathrm{Sg} \otimes_{\mathrm{Sh}^{W}}\left(\mathrm{Sh}^{W} \otimes H^{\bullet}(\mathcal{B})\right)=\mathbb{C}\left[\mathfrak{g}^{*}\right] \otimes H^{\bullet}(\mathcal{B})
$$

is a finite rank free $\mathbb{C}\left[\mathfrak{g}^{*}\right]$-module. Therefore, this $\mathbb{C}\left[\mathfrak{g}^{*}\right]$-module has no torsion submodules and we conclude that the map $\psi^{*}$ is injective.

To prove surjectivity, we let the group $\mathbb{C}^{\times}$act on the vector space $\mathfrak{g} \times \mathfrak{h}$, resp. on $\widetilde{\mathfrak{g}}^{*}$, by dilations. This makes the map $\psi: \widetilde{\mathfrak{g}}^{*} \rightarrow \mathfrak{g}^{*} \otimes_{\mathfrak{h}^{*} / W} \mathfrak{h}^{*}$ a $\mathbb{C}^{\times}$-equivariant morphism of $\mathbb{C}^{\times}$-varieties. It follows that $\psi^{*}$, the pull-back map, is an injective degree preserving map of the corresponding coordinate rings. Comparing formulas (4.2.2) and (4.2.3) we see that, for each degree, the corresponding homogeneous components of $\mathrm{Sg} \otimes_{\mathrm{Sh}^{W}} \mathrm{Sh}$ and $\Gamma\left(\widetilde{\mathfrak{g}}^{*}, \mathcal{O}_{\widetilde{\mathfrak{g}}^{*}}\right)$ have equal dimensions. Thus, the map $\psi^{*}$ must be an isomorphism.

Remarks 4.2.4. (i) Let $\mathfrak{g}^{\text {reg }} \subset \mathfrak{g}^{*}$ be the union of Ad $G$-conjugacy classes in $\mathfrak{g}^{*}$ of maximal dimension, and put $\widetilde{\mathfrak{g}}^{\text {reg }}:=\mu^{-1}\left(\mathfrak{g}^{\text {reg }}\right)$. We claim that the restriction of the morphism $\psi$ in 
(4.1.3) to $\widetilde{\mathfrak{g}}^{\text {reg }}$ yields an isomorphism of algebraic varieties

$$
\psi^{\text {reg }}: \widetilde{\mathfrak{g}}^{\mathrm{reg}} \stackrel{\sim}{\longrightarrow} \mathfrak{g}^{\mathrm{reg}} \times_{\mathfrak{h}^{*} / W} \mathfrak{h}^{*} .
$$

To see this, one verifies first, using Jordan decomposition in $\mathfrak{g}$, that the map $\mu^{\text {reg }}$ : $\widetilde{\mathfrak{g}}^{\text {reg }} \rightarrow \mathfrak{g}^{\text {reg }}$ has finite fibers. It follows, since $\mathfrak{h}^{*}$ is finite over $\mathfrak{h}^{*} / W$, that the map $\psi^{\text {reg }}$ has finite fibers as well. Furthermore, using that $\psi^{\text {reg }}$ is also proper, we deduce that it is, in fact, a finite dominant morphism between smooth schemes. Moreover, it is clear that the map $\psi^{\text {reg }}$ is one-to-one over the Zariski open subset of semisimple regular elements of $\mathfrak{g}$. Therefore, such a morphism must automatically be an isomorphism.

(ii) Kostant showed that $\varpi^{-1}(0)$, the scheme-theoretic zero fiber of the morphism $\varpi$, is reduced at any point of $\varpi^{-1}(0) \cap \mathfrak{g}^{\text {reg }}$. To prove this, he verified by a direct computation that the generators of $\mathbb{C}[\mathfrak{g}]^{G}$ have linearly independent differentials at any point of $\mathfrak{g}^{\text {reg, }}$ see [Ko2], and also [CG, §6.7] for a slightly different argument.

Here is an alternate argument which involves no computation and is independent of Ko2. By Remark (i), we may identify $\nu: \widetilde{\mathfrak{g}}^{\text {reg }} \rightarrow \mathfrak{h}^{*}$, the restriction of the smooth morphism $\nu$ to $\mathfrak{g}^{\text {reg }}$, with the projection $\mathfrak{g}^{\text {reg }} \times_{\mathfrak{h}^{*} / W} \mathfrak{h}^{*} \rightarrow \mathfrak{h}^{*}$. Applying to this projection base change with respect to the flat map $\mathfrak{h}^{*} \rightarrow \mathfrak{h}^{*} / W$ in diagram (4.1.2), we deduce that the morphism $\varpi: \mathfrak{g}^{\text {reg }} \rightarrow \mathfrak{h}^{*} / W$ is also smooth. Hence its zero-fiber is reduced.

\section{References}

[ABG] S. Arkhipov, R. Bezrukavnikov, V. Ginzburg, Quantum groups, the loop Grassmannian, and the Springer resolution. J. Amer. Math. Soc. 17 (2004), 595-678.

[AC] E. Akyildiz, J. Carrell, Cohomology of projective varieties with regular $S L_{2}$-actions. Manuscripta Math. 58 (1987), 473-486.

[BBD] A. Beilinson, J. Bernstein, P. Deligne, Faisceaux pervers. Astérisque 100 (1982).

[BBM] W. Borho, J.-L. Brylinski, R. MacPherson, Nilpotent orbits, primitive ideals, and characteristic classes. Progress in Math., 78. Birkhüser Boston, 1989.

[BD] A. Beilinson, V. Drinfeld, Quantization of Hitchin's integrable system and Hecke eigensheaves. Available at http://www.math.uchicago.edu/〜mitya/langlands.

[BL1] J. Bernstein, V. Lunts, Equivariant sheaves and functors. Lecture Notes in Mathem., 1578. Springer-Verlag, Berlin, 1994.

[BL2] J. Bernstein, V. Lunts, A simple proof of Kostant's theorem that $U(\mathfrak{g})$ is free over its center. Amer. J. Math. 118 (1996), 979-987.

[BFM] R. Bezrukavnikov, M. Finkelberg, I. Mirković, Equivariant homology and K-theory of affine Grassmannians and Toda lattices. Compos. Math. 141 (2005), 746-768.

[BB] A. Bialynicki-Birula, Some theorems on actions of algebraic groups. Ann. of Math. 98 (1973), 480.

[Bro] A. Broer, Lectures on decomposition classes. Representation theories and algebraic geometry (Montreal, PQ, 1997), 39-83, NATO Adv. Sci. Inst. Ser. C Math. Phys. Sci., 514, Kluwer, 1998.

[Bry] R. Brylinski, Limits of weight spaces, Lusztig's q-analogs, and fiberings of adjoint orbits. J. Amer. Math. Soc. 2 (1989), 517-533.

[CG] N. Chriss, V. Ginzburg, Representation Theory and Complex Geometry. Birkhäuser Boston, 1997.

[De] P. Deligne, La conjecture de Weil. II. I.H.E.S. Publ. Math. 52 (1980), 137-252.

[FM] R. Friedman, J. Morgan, Minuscule representations, invariant polynomials, and spectral covers. Vector bundles and representation theory (Columbia, MO, 2002), 1-41, Contemp. Math., 322, Amer. Math. Soc., Providence, RI, 2003. arXiv:math.AG/0108104. 
[G1] V. Ginzburg, Perverse sheaves on a loop group and Langlands' duality. arXiv:alg-geom/9511007.

[G2] V. Ginzburg, Perverse sheaves and $\mathbb{C}^{*}$-actions. J. Amer. Math. Soc. 4 (1991), 483-490.

[GK] V. Ginzburg, S. Kumar, Cohomology of quantum groups at roots of unity. Duke Math. J. 69 (1993), 179-198.

[GKM] M. Goresky, R. Kottwitz, R. MacPherson, Equivariant cohomology, Koszul duality, and the localization theorem. Invent. Math. 131 (1998), 25-83.

[Gr] B. Gross, On Minuscule Representations and the Principal SL $L_{2}$. Repr. Theory 4 (2000), 225- 244.

[KL] D. Kazhdan, G. Lusztig, Schubert varieties and Poincar duality. Geometry of the Laplace operator. Proc. Sympos. Pure Math., XXXVI, pp. 185-203, Amer. Math. Soc. 1980.

[Ko1] B. Kostant, Lie group representations on polynomial rings, Amer. J. Math., 85 (1963), 327-404.

[Ko2] B. Kostant, The principal three-dimensional subgroup and the Betti numbers of a complex simple Lie group. Amer. J. Math., 81 (1959), 973-1032.

[Ko3] B. Kostant, On Whittaker vectors and representation theory. Invent. Math. 48 (1978), $101-184$.

[Lu] G. Lusztig, Singularities, Character formulas and a q-analog of weight multiplicity. Asterisque $101-102$ (1983), 208-229.

[MV] I. Mirković, K. Vilonen, Perverse sheaves on affine Grassmannians and Langlands duality. Math. Res. Lett. 7 (2000), 13-24.

[Se] J.-P. Serre, Corps locaux. Hermann, Paris 1962.

Department of Mathematics, University of Chicago, Chicago IL 60637, USA;

ginzburg@math.uchicago.edu 